\title{
Diagnostic and prognostic role of BTA, NMP22, survivin and cytology in urothelial carcinoma
}

\author{
Yu-Wen Gong ${ }^{1 \#}$, Yi-Ran Wang ${ }^{1 \#}$, Guang-Rui Fan ${ }^{1}$, Qian Niu ${ }^{1}$, You-Li Zhao ${ }^{1}$, Hanzhang Wang ${ }^{2}$, \\ Robert Svatek $^{2}$, Ronald Rodriguez ${ }^{2}$, Zhi-Ping Wang ${ }^{1 \wedge}$ \\ ${ }^{1}$ Institute of Gansu Nephro-Urological Clinical Center, Department of Urology, Institute of Urology, Key Laboratory of Urological Disease of \\ Gansu Province, Lanzhou University Second Hospital, Lanzhou, China; ${ }^{2}$ Department of Urology, The University of Texas Health Science Center at \\ San Antonio, San Antonio, Texas 78229, USA \\ Contributions: (I) Conception and design: YW Gong; (II) Administrative support: ZP Wang; (III) Provision of study materials or patients: YR Wang; \\ (IV) Collection and assembly of data: YR Wang, GR Fan, YL Zhao; (V) Data analysis and interpretation: YW Gong, Q Niu, H Wang, R Svatek, R \\ Rodriguez; (VI) Manuscript writing: All authors; (VII) Final approval of manuscript: All authors. \\ \#These authors contributed equally to this work. \\ Correspondence to: Zhi-Ping Wang. 82 Cuiyingmen, Lanzhou 730000, China. Email: wangzplzu@163.com.
}

Background: Cytology is a recommended noninvasive urine test for the detection and surveillance of bladder cancer and upper-tract urothelial carcinoma. It is however characterized by poor sensitivity in lowgrade tumors. This study aims to determine the diagnostic and prognostic role of BTA, BTA-stat, NMP22, and Survivin.

Methods: Urine samples were collected from a total of 105 patients (bladder cancer ( $\mathrm{n}=61$ ), upper-tract urothelial carcinoma $(n=44)$, and controls $(n=52)$. The samples were directly assessed using cytology, BTAstat (Qualitative test), BTA (chemiluminescence test), NMP22 (Qualitative test), and Survivin (enzymelinked immunosorbent assay). Cancer progression and recurrence were assessed after a median follow-up of 32 months (4-47 months). Univariate and multivariate analyses were performed using Kaplan-Meier survival analysis and Cox proportional hazards regression.

Results: The triple combination of Survivin + BTA + Cytology was the most promising model for discriminating bladder cancer or upper-tract urothelial carcinoma from controls (UTUC group: the area under the curve value 0.97 , sensitivity $86 \%$, specificity $96 \%$; BC group: the area under the curve value 0.86 sensitivity $67 \%$, specificity $96 \%)$. Univariate survival analysis, showed Cytology $(\mathrm{P}=0.02 ; \mathrm{HR}=5.35)$ and Survivin $(\mathrm{HR}=3.24 ; \mathrm{P}=0.03)$ to have a significant association with the progression-free survival, while Survivin $(\mathrm{HR}=4.15 ; \mathrm{P}=0.04)$ was statistically associated with cancer-specific survival in the bladder cancer group. The multivariable analysis did not show any of these markers as independent prognostic factors.

Conclusions: These biomarkers showed a higher sensitivity than cytology, but a poorer specificity. All biomarkers exhibited good diagnostic performance in both bladder cancer and upper-tract urothelial carcinoma. Combining Survivin + BTA + Cytology was superior to the use of a single marker or combining other biomarkers.

Keywords: Biomarkers; urothelial carcinoma (UC); combined detection; diagnosis; prognosis

Submitted Mar 02, 2021. Accepted for publication Jun 25, 2021.

doi: $10.21037 /$ tcr-21-386

View this article at: https://dx.doi.org/10.21037/tcr-21-386

^ ORCID: Yu-Wen Gong, 0000-0003-3134-5696; Zhi-Ping Wang, 0000-0002-8140-591X. 


\section{Introduction}

Urothelial carcinomas (UCs), are the fourth most common tumors and mainly include bladder cancers (BC) and upper tract urothelial carcinomas (UTUC) (1). BC is the most common urinary tract malignancy accounting for $90-95 \%$ of UCs while UTUC is a rare lethal disease accounting for approximately $5 \%$ of UCs (2).

Approximately $75 \%$ of patients with BC, are initially diagnosed with non-muscle-invasive bladder cancer (NMIBC) (3). Although these lesions are not immediately life-threatening, they have a high probability of recurrence (up to $50 \%$ ) and progression (up to 20\%) (4). Despite UTUC and BC sharing many characteristics, they are two distinct diseases $(5,6) .60 \%$ of patients with UTUC have an invasive disease while $7 \%$ have metastasis on the first diagnosis. Cancer recurrence in the bladder occurs in $22-47 \%$ of UTUC patients compared with $2-6 \%$ recurrence in the contralateral upper tract following surgery $(2,7)$. Therefore, early diagnosis and stringent follow-up are crucial.

The methods currently used in the detection and surveillance of BC and UTUC include endoscopic examination (cystoscopy or ureteroscopy), imaging and urine cytology. Cytology is recommended as a noninvasive urine test due to high specificity. Urine cytology is unreliable as it has poor sensitivity in low-grade BC (16\%) (8), cytological interpretation is highly observer-dependent and evaluation is hampered by low cellular yield, urinary tract infections, stones, or intravesical installations (3). The U.S. Food and Drug Administration (FDA) approved FISH, Immunocyt, BTA, and NMP22 as auxiliary testing methods for BC. However, these markers showed low specificity and only a few studies have investigated these biomarkers in the Chinese population. These biomarkers have also not been recommended by guidelines for UTUC diagnosis. However, some biomarkers, such as E-cadherin, HER2, and Ki-67 have been mentioned in guidelines for UTUC prognosis $(9,10)$. Studies done to evaluate the prognostic and diagnostic role of the biomarkers have limitations of retrospective design and small sample size. Therefore, numerous urinary tests have been developed for the diagnosis and surveillance of BC. In this study, cytology was combined with BTA-stat, quantitative BTA, NMP22, and Survivin in a prospective analysis of prognostic and diagnostic tests in BC and UTUC.

We present the following article in accordance with the REMARK reporting checklist (available at https://dx.doi. org/10.21037/tcr-21-386).

\section{Methods}

\section{Patient cohort and sample}

The study was conducted in accordance with the Declaration of Helsinki (as revised in 2013) and approved by the Ethics Committee of Lanzhou University Second Hospital (approval number: 2016A-058). Written informed consent was obtained from all participants before the samples were collected. A total of 157 cases of the UC group (BC group, UTUC group) and control group (healthy individuals and patients with other urinary diseases) were included in this study carried out between October 2015 and May 2017.

About $10 \mathrm{~mL}$ of the first-morning urine was collected from each individual for the detection of BTA, BTA-STAT, NMP22, and Survivin. The entire urine volume of the second voided morning urine from both study groups was then collected for cytological examinations. Collected data variables included: patient demographics, date of the urine collection, surgical pathological examination, recurrence, and follow-up. Inclusion criteria: The patients in the UC group were diagnosed based on histological examination while the healthy individuals (normal medical reports) underwent a physical examination in the Physical Examination Center affiliated to Lanzhou University second hospital and patients with other urinary diseases were recruited from the Department of Urology, Lanzhou University second hospital. Exclusion criteria: patients who had undergone invasive examination, patients who had suffered from both UTUC and $\mathrm{BC}$, patients with incomplete clinical information, and patients who declined to sign the informed consent.

\section{Urine detection}

\section{Cytology}

Urine cytology was examined in the Department of Pathology in Lanzhou University second hospital with Thinprep cytologic test (TCT) and interpreted by two experienced pathologists. The cytopathological findings were classified as negative or positive, in correspondence to the Papanicolaou classes I-III and IV-V, respectively.

\section{NMP22 and BTA-stat}

Both the NMP22 (Alere Inc. USA) and BTA-stat assays (Polymedco CDP, LLC. USA) were point-of-care devices. The assay was carried out by dripping the urine into the device and reading the results after 30 minutes in the NMP22 test and after 5 minutes in the BTA-stat test. Both results were interpreted by a single observer who was 
blinded to the result of various clinical examination.

\section{Quantitative BTA and surviving}

Urine samples were centrifuged as soon as possible at 3,000 rpm, $4{ }^{\circ} \mathrm{C}$, for 10 minutes. The supernatant was collected and stored at $-80^{\circ} \mathrm{C}$. All samples were brought to room temperature before carrying out chemiluminescence for quantitative BTA (Pergrande, Beijing, China), and enzyme-linked immunosorbent assay (ELISA) for Survivin (R\&D systems, MN, USA).

\section{Follow-up}

To analyze the prognostic performance, tumor recurrence-free survival (RFS), progression-free survival (PFS), and cancerspecific survival (CSS) were determined. Recurrence was defined to have occurred if patients presented with the same grade and stage as the previous tumor, or lower stage or grade than the previous tumor, or presented with a non-muscle invasive tumor in the bladder in UTUC patients. Progression was said to have occurred if patients presented with a higher grade or stage than the recurrent tumor, metastasis, or with a muscle-invasive tumor in the bladder in UTUC patients.

\section{Statistical calculations}

Statistical analysis was performed using SPSS version 19.0 and GraphPad prism7. The diagnostic performance of these markers was determined using receiver operating characteristic (ROC) curves and the area under the curve (AUC) values. The optimal cutoff values for the single markers were determined by calculation of the Youden index. In combination tests, both parallel and serial tests were analyzed. The Chi-square test was used to analyze differences between two classified variable groups while the Mann-Whitney $U$ test was used for independent samples with a continuous variable. Recurrence, progression, and death risk were evaluated by the Kaplan-Meier method, with the log-rank test, and multivariable analysis with Cox proportional hazards models. All tests were two-sided, and $\mathrm{P}$ values $<0.05$ were considered statistically significant.

\section{Results}

\section{Patient characteristics}

The median age of patients with UCs was 70 (60-76) years old, with males accounting for about $69 \%$ and females $31 \%$. A total of 105 participants were included in the study. The stage and grade of 61 patients with $\mathrm{BC}$ were as follows: 30 NMIBC low grade (LG), 15 NMIBC high grade (HG), and 16 MIBC HG. UTUC included 22 patients with renal pelvic cancer, 14 patients with ureter cancer, and 8 patients with both renal pelvic cancer and ureter cancer. The stage and grade of 44 patients with UTUC were as follows: 7 noninvasive UTUC LG, 7 non-invasive UTUC HG, 1 flat lesion, 8 invasive carcinoma LG, and 21 invasive carcinoma HG. The control group ( $\mathrm{n}=52$ ) consisted of 32 patients with other urinary diseases including benign prostate hyperplasia, urinary tract infection, hematuria, lithiasis, and 20 healthy volunteers. Their clinicopathological data are summarized in Table 1.

The median follow-up period for BC patients and UTUC patients was 32 months (4-47 months). During the follow-up, 4 patients in the BC group and 3 patients in the UTUC group were lost to follow-up. Four patients experienced local tumor recurrence, 15 patients experienced progression and 10 patients died due to cancer-related events in the $\mathrm{BC}$ group. Two patients experienced low-grade BC, 13 patients had cancer progression, and 7 patients died due to cancer-related events in the UTUC group.

\section{Single test performance}

The AUCs of BTA, BTA-stat, NMP22, Survivin and cytology for differentiating BC from the control group were $0.84,0.67,0.69,0.84$ and 0.59 , respectively. Sensitivity values of each marker for BC were as follows: C, 18\% (3\% for $\mathrm{LG}$ and $37 \%$ for HG, $11 \%$ for NMIBC and $37 \%$ for MIBC); Bs, $69 \%$ (50\% for LG and 93\% for HG; $55 \%$ for NMIBC and $95 \%$ for MIBC); N, 59\% (35\% for LG and $89 \%$ for $\mathrm{HG}$; $53 \%$ for NMIBC and $74 \%$ for MIBC); and B, $74 \%$ (56\% for LG and $96 \%$ for HG; 66\% for NMIBC and $95 \%$ for MIBC); S, $77 \%$ (59\% for LG and $96 \%$ for $\mathrm{HG}$; $66 \%$ for NMIBC and $95 \%$ for MIBC).

The AUCs of BTA, BTA-stat, NMP22, Survivin and cytology for differentiating UTUC from control group were $0.93,0.81,0.76,0.88$ and 0.65 , respectively. Sensitivity values of each marker for UTUC were as follows: C, $30 \%$ (13\% for LG and 38\% for HG; $31 \%$ for TaT 1 tumor and $28 \%$ for T2-T4 tumor); Bs, $98 \%$ (93\% for LG and $100 \%$ for HG; $100 \%$ for TaT1 tumor and $97 \%$ for T2-T4 tumor); N, 73\% (60\% for LG and 79\% for HG; $54 \%$ for TaT1 tumor and $79 \%$ for T2-T4 tumor); and B, 95\% (87\% for LG and 97\% for HG; $92 \%$ for TaT1 tumor and 94\% for T2-T4 tumor); S, 89\% (87\% for LG and 90\% for HG; $85 \%$ for TaT 1 tumor and $90 \%$ for T2-T4 tumor). 
Table 1 Demographic and clinicopathological characteristics of study subjects

\begin{tabular}{|c|c|}
\hline Group & $\mathrm{N}(\%)$ \\
\hline UCs & $105(67)$ \\
\hline \multicolumn{2}{|l|}{ Sex } \\
\hline Male & $73(70)$ \\
\hline Female & $32(30)$ \\
\hline \multicolumn{2}{|l|}{$\mathrm{BC}$} \\
\hline \multicolumn{2}{|l|}{ History of UC } \\
\hline Primary & $49(80)$ \\
\hline Recurrence & $12(20)$ \\
\hline \multicolumn{2}{|l|}{ Grade } \\
\hline PUNLMP & $3(5)$ \\
\hline LG & $31(51)$ \\
\hline$H G$ & $27(44)$ \\
\hline \multicolumn{2}{|l|}{ Stage } \\
\hline Ta-T1 & $47(77)$ \\
\hline T2-Т4 & $14(23)$ \\
\hline \multicolumn{2}{|l|}{ UTUC } \\
\hline \multicolumn{2}{|l|}{ History of BC } \\
\hline No & $43(98)$ \\
\hline Yes & $1(2)$ \\
\hline \multicolumn{2}{|l|}{ Location } \\
\hline Renal pelvis & $22(50)$ \\
\hline Ureter & $14(32)$ \\
\hline Pelvis and ureter & $8(18)$ \\
\hline \multicolumn{2}{|l|}{ Grade } \\
\hline$L G$ & $15(34)$ \\
\hline$H G$ & $29(66)$ \\
\hline \multicolumn{2}{|l|}{ Stage } \\
\hline Tis only & $1(2)$ \\
\hline Ta-T1 & 14 (32) \\
\hline T2-Т4 & $29(66)$ \\
\hline Control & $52(33)$ \\
\hline \multicolumn{2}{|l|}{ Sex } \\
\hline Male & $35(67)$ \\
\hline Female & 17 (33) \\
\hline
\end{tabular}

Table 1 (continued)
Table 1 (continued)

\begin{tabular}{|c|c|}
\hline Group & $\mathrm{N}(\%)$ \\
\hline Other disease & $32(62)$ \\
\hline Healthy & 20 (38) \\
\hline \multicolumn{2}{|c|}{$\begin{array}{l}\text { UCs, urothelial carcinomas; BC, bladder cancer; UTUC, } \\
\text { upper-tract urothelial carcinoma; PUNLMP, papillary urothelia } \\
\text { neoplasm of low malignant potential; LG, low grade; HG, } \\
\text { high grade; Tis, tumor in situ. Grade according to } 2004 \text { WHO } \\
\text { classification; Stage according to } 2009 \text { TNM classification; }\end{array}$} \\
\hline
\end{tabular}

Specificity results of each marker were as follows: C, $100 \%$; Bs, 63\%; N, 79\%; B, 81\%; and S, 83\% (Figure 1, Table 2). The cut-off of BTA and Survivin as quantitative tests were $88 \mathrm{U} / \mathrm{mL}$ and $21.8 \mathrm{pg} / \mathrm{mL}$, respectively (Figure 1 , Table 2).

\section{Combination test performance}

A combination of urinary markers with cytology, a total of 15 combined models can be generated $(\mathrm{B}+\mathrm{C}, \mathrm{Bs}+\mathrm{C}$, $\mathrm{S}+\mathrm{C}, \mathrm{N}+\mathrm{C}, \mathrm{B}+\mathrm{S}+\mathrm{C}, \mathrm{BS}+\mathrm{S}+\mathrm{C}, \mathrm{S}+\mathrm{N}+\mathrm{C}, \mathrm{B}+\mathrm{N}+\mathrm{C}, \mathrm{B}+\mathrm{BS}+\mathrm{C}$, $\mathrm{BS}+\mathrm{N}+\mathrm{C}, \mathrm{B}+\mathrm{BS}+\mathrm{N}+\mathrm{C}, \mathrm{BS}+\mathrm{S}+\mathrm{N}+\mathrm{C}, \mathrm{B}+\mathrm{S}+\mathrm{N}+\mathrm{C}, \mathrm{BS}+\mathrm{B}+\mathrm{S}+\mathrm{C}$, $\mathrm{BS}+\mathrm{B}+\mathrm{S}+\mathrm{N}+\mathrm{C}$ ). Among the combination of a single tumor marker with $\mathrm{C}$, the $\mathrm{B}+\mathrm{C}$ yielded the best diagnostic performance in UTUC group (AUC 0.91, sensitivity $96 \%$, specificity $81 \%$, PPV $81 \%$, NPV 95\%); while $\mathrm{S}+\mathrm{C}$ had the best performance in BC group (AUC 0.81, sensitivity $77 \%$, specificity $83 \%$, PPV $84 \%$, NPV $75 \%$ ). After comprehensive consideration, the triple combination of $\mathrm{S}+\mathrm{B}+\mathrm{C}$ was the most promising models in all models, with cost-savings, and valuable in both UTUC group and BC group (UTUC group: AUC 0.97 , sensitivity $86 \%$, specificity 96\%, PPV 95\%, NPV 89\%; BC group: AUC 0.86 , sensitivity $67 \%$, specificity $96 \%$, PPV $95 \%$, NPV 78\%) (Figure 1, Table 3).

\section{Associations of the urinary markers and cytology with clinical parameters}

In the $\mathrm{BC}$ group, cytology $(\mathrm{P}=0.02), \mathrm{BTA}$-stat $(\mathrm{P}=0.017)$, and BTA $(\mathrm{P}=0.001)$ were statistically associated with tumor stage and all these markers included cytology were statistically associated with tumor grade (Table 4). Tumor recurrence and size $\geq 3 \mathrm{~cm}$ had a significant correlation to a positive test of NMP22 and BTA-stat, respectively. In the UTUC group, only the expression of Survivin had a 

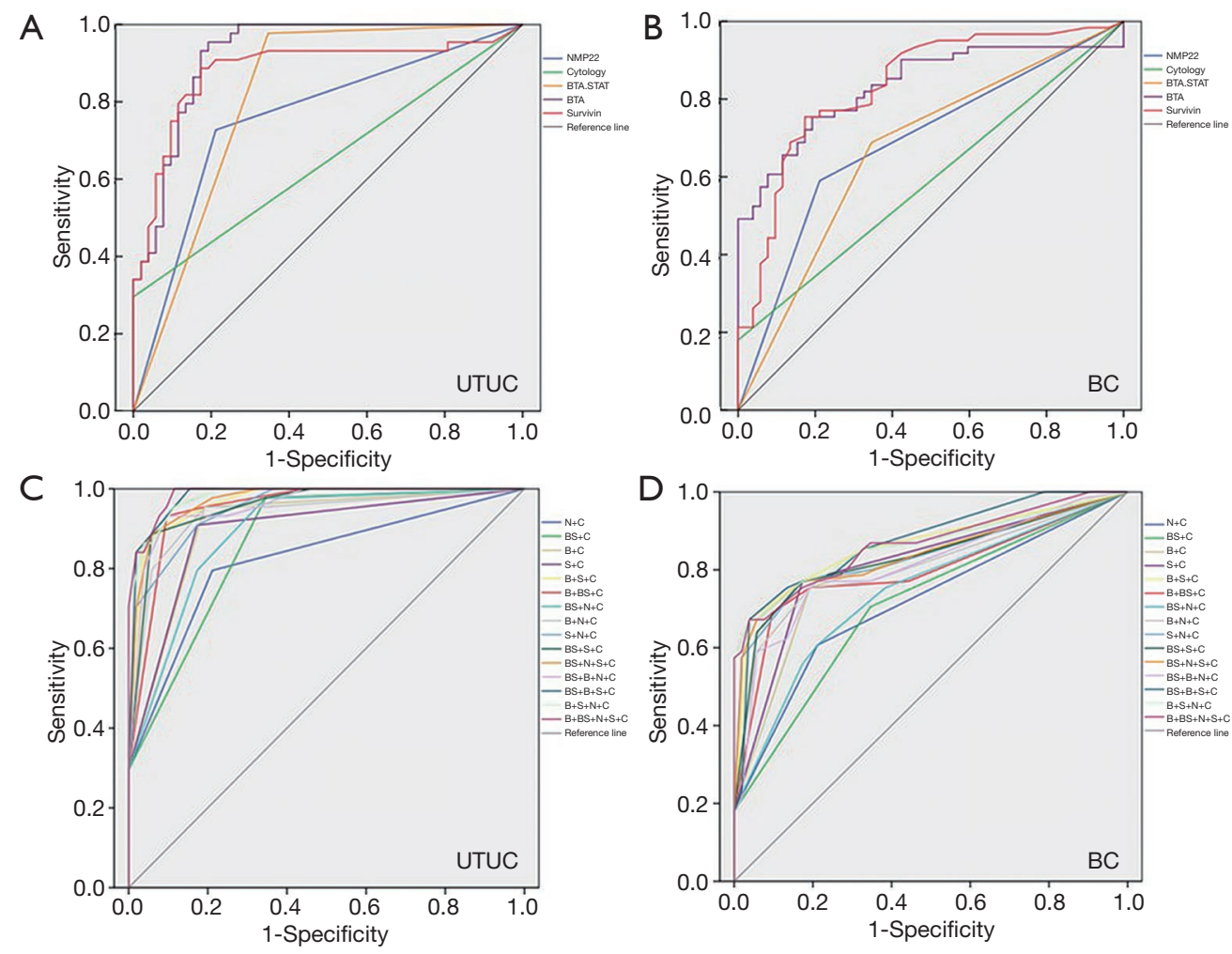

Figure 1 Diagnostic performance of single urine biomarker and combinations. (A) ROC for single urine tests in UTUC. (B) ROC for single urine tests in BC. (C) ROC for different combinations of cytology and other biomarkers in UTUC. (D) ROC for different combinations of cytology and other biomarkers in BC. Youden Index cutoffs that maximized sum of sensitivity and specificity were determined for each test. ROC, area under the curve; UTUC, upper-tract urothelial carcinoma; BC, bladder cancer.

significant correlation to a higher tumor stage (Table 4).

\section{Survival prediction}

We developed the Kaplan-Meier survival analysis of each urinary biomarker and cytology (Figures 2-4). Cytology $(\mathrm{P}=0.02)$, and Survivin $(\mathrm{P}=0.03)$ had a significant association with PFS, and Cytology ( $\mathrm{P}=0.03)$, Survivin $(\mathrm{P}=0.04)$ was also statistically associated with CSS in the BC group. None of the markers showed a significant association to the PFS or CSS in the UTUC group. (There were too few recurrence cases to analyze the relationship between the urine markers and recurrence-free survival).

We also analyzed the predicting performance of clinicopathological parameters with PSF and CSS. The univariate analysis predicted poor prognosis with a higher stage $(\mathrm{P}=5.75 \mathrm{E}-05)$, and HG $(\mathrm{P}=2.08 \mathrm{E}-04)$ in the $\mathrm{BC}$ group. In the UTUC group, tumor number $\geq 2$ had a significantly increased risk of tumor progression $(\mathrm{P}=0.02)$, while the tumor grade showed a significant association with CSS $(\mathrm{P}=0.03)$. Multivariate analysis revealed that the tumor stage was an independent prognostic factor for both PFS and CSS $(\mathrm{P}=0.03 ; \mathrm{P}=0.004)$, while tumor number $\geq 2$ was an independent prognostic factor for CSS $(\mathrm{P}=0.01)$ in the $\mathrm{BC}$ group. And the tumor grade was an independent prognostic factor for CSS $(\mathrm{P}=0.04)$. However, none of these biomarkers was an independent prognostic factor in $\mathrm{BC}$ or UTUC (Tables 5,6).

\section{Discussion}

In this study, both UTUC and BC samples were included for the detection of BTA, BTA-stat, NMP22, Survivin, and cytology. The results revealed that all tumor markers and urine cytology were effective in the diagnosis of both BC and UTUC, with superior effectiveness in the UTUC group. Urine cytology is recommended as an auxiliary examination. However, it is plagued by low sensitivity 
Table 2 Test results of single urinary tests in UTUC and BC group

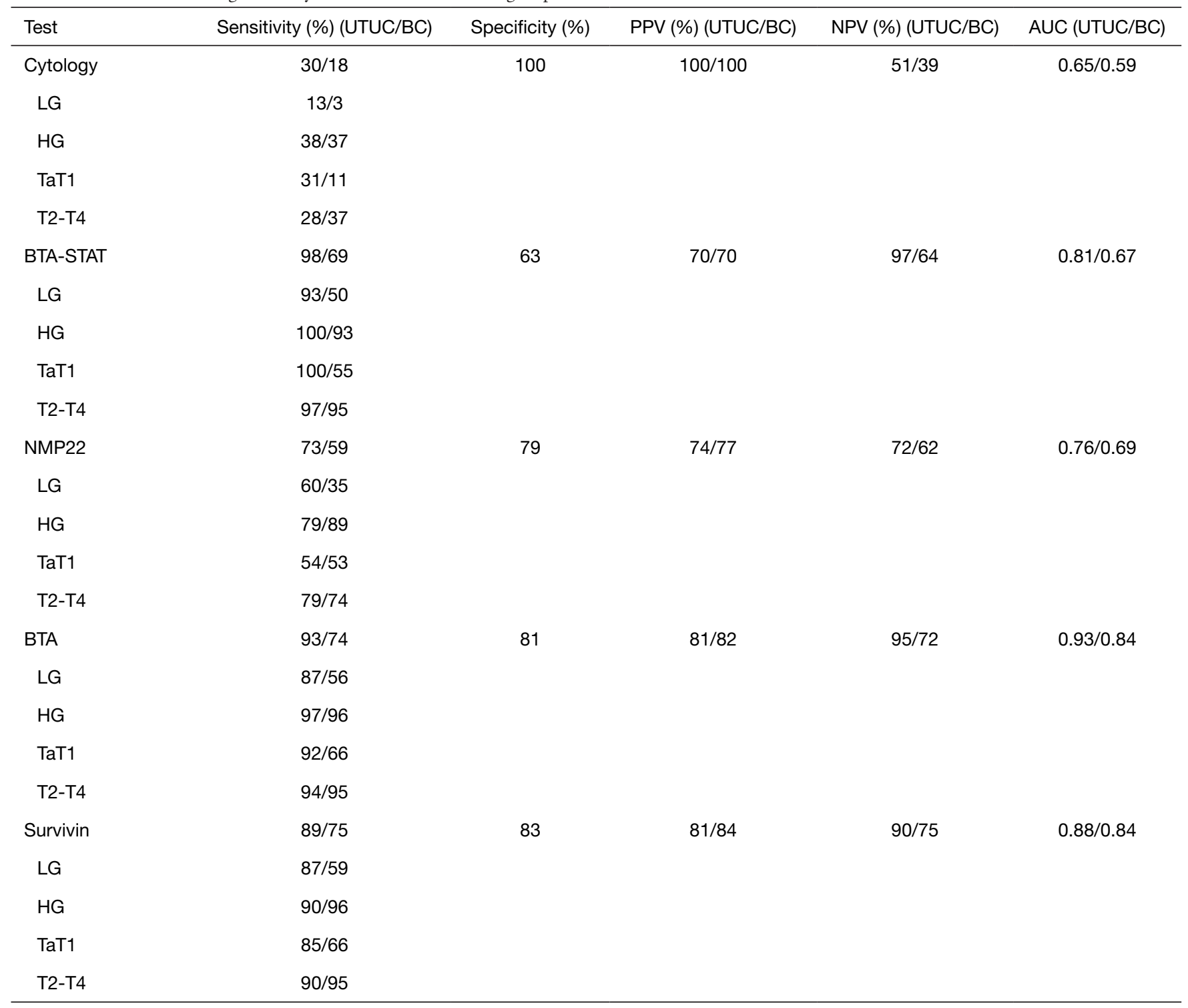

LG, low grade; HG, high grade; BC, bladder cancer; UTUC, upper-tract urothelial carcinoma; AUC, area under the curve; NPV, negative predictive value; PPV, positive predictive value. Grade according to 2004 WHO classification; Stage according to 2009 TNM classification.

in detecting low-grade noninvasive lesions, and lack of standardized diagnostic criteria. In 2016, a standardized reporting system redefining urinary cytology diagnostic categories was published by the Paris Working Group (11). Since patients were enrolled in this study before 2015 before the publication of the Paris Working Group, the cytology results were classified according to the Papanicolaou classes. This study revealed that cytology has a high specificity, but low sensitivity $18 \%$, especially for low-grade bladder tumors. These results were consistent with similar reported findings (12). Surprisingly, the sensitivity of the cytology test was higher in UTUC than BC group and was correlated with the tumor grade, which was consistent with earlier reports $(13,14)$.

BTA is a "bladder tumor antigen" human complement factor H-related protein (15). In this study we performed both qualitative and quantitative tests. The AUC of the BTA qualitative test for $\mathrm{BC}$ was 0.67 , and the diagnostic sensitivity and specificity were $69 \%$ and $63 \%$, respectively. These results revealed a higher sensitivity and a lower 
Table 3 Test results of combinations of cytology and other urinary tests in UTUC and BC group

\begin{tabular}{|c|c|c|c|c|c|}
\hline The combination tests & Sensitivity (\%) (UTUC/BC) & Specificity (\%) & PPV (\%) (UTUC/BC) & NPV (\%) (UTUC/BC) & AUC (UTUC/BC) \\
\hline $\mathrm{N}+\mathrm{C}$ & $80 / 61$ & 79 & $76 / 77$ & $82 / 63$ & $0.82 / 0.72$ \\
\hline $\mathrm{BS}+\mathrm{C}$ & $98 / 71$ & 65 & $71 / 71$ & $97 / 65$ & $0.87 / 0.71$ \\
\hline $\mathrm{S}+\mathrm{C}$ & $91 / 77$ & 83 & $82 / 84$ & $91 / 75$ & $0.90 / 0.81$ \\
\hline$B+S+C$ & $86 / 67$ & 96 & $95 / 95$ & $89 / 78$ & $0.97 / 0.86$ \\
\hline $\mathrm{BS}+\mathrm{N}+\mathrm{C}$ & $80 / 56$ & 83 & $80 / 79$ & $83 / 61$ & $0.89 / 0.74$ \\
\hline $\mathrm{B}+\mathrm{N}+\mathrm{C}$ & $80 / 75$ & 81 & $81 / 82$ & $95 / 74$ & $0.94 / 0.81$ \\
\hline $\mathrm{S}+\mathrm{N}+\mathrm{C}$ & $91 / 77$ & 83 & $82 / 84$ & $91 / 75$ & $0.95 / 0.83$ \\
\hline $\mathrm{BS}+\mathrm{S}+\mathrm{C}$ & $89 / 64$ & 94 & $93 / 93$ & $91 / 69$ & $0.96 / 0.83$ \\
\hline$B S+B+S+C$ & $84 / 61$ & 98 & $97 / 97$ & $88 / 68$ & $0.98 / 0.87$ \\
\hline $\mathrm{B}+\mathrm{S}+\mathrm{N}+\mathrm{C}$ & $70 / 57$ & 100 & $100 / 100$ & $80 / 67$ & $0.98 / 0.86$ \\
\hline $\mathrm{B}+\mathrm{BS}+\mathrm{N}+\mathrm{S}+\mathrm{C}$ & $93 / 67$ & 92 & $91 / 91$ & $94 / 71$ & $0.98 / 0.87$ \\
\hline
\end{tabular}

N, NMP22; BS, BTA-BTAT; S, Survivin; B, BTA; C, cytology; AUC, area under the curve; NPV, negative predictive value; PPV, positive predictive value.

Table 4 Association of clinical parameters with urinary markers in UTUC and BC group

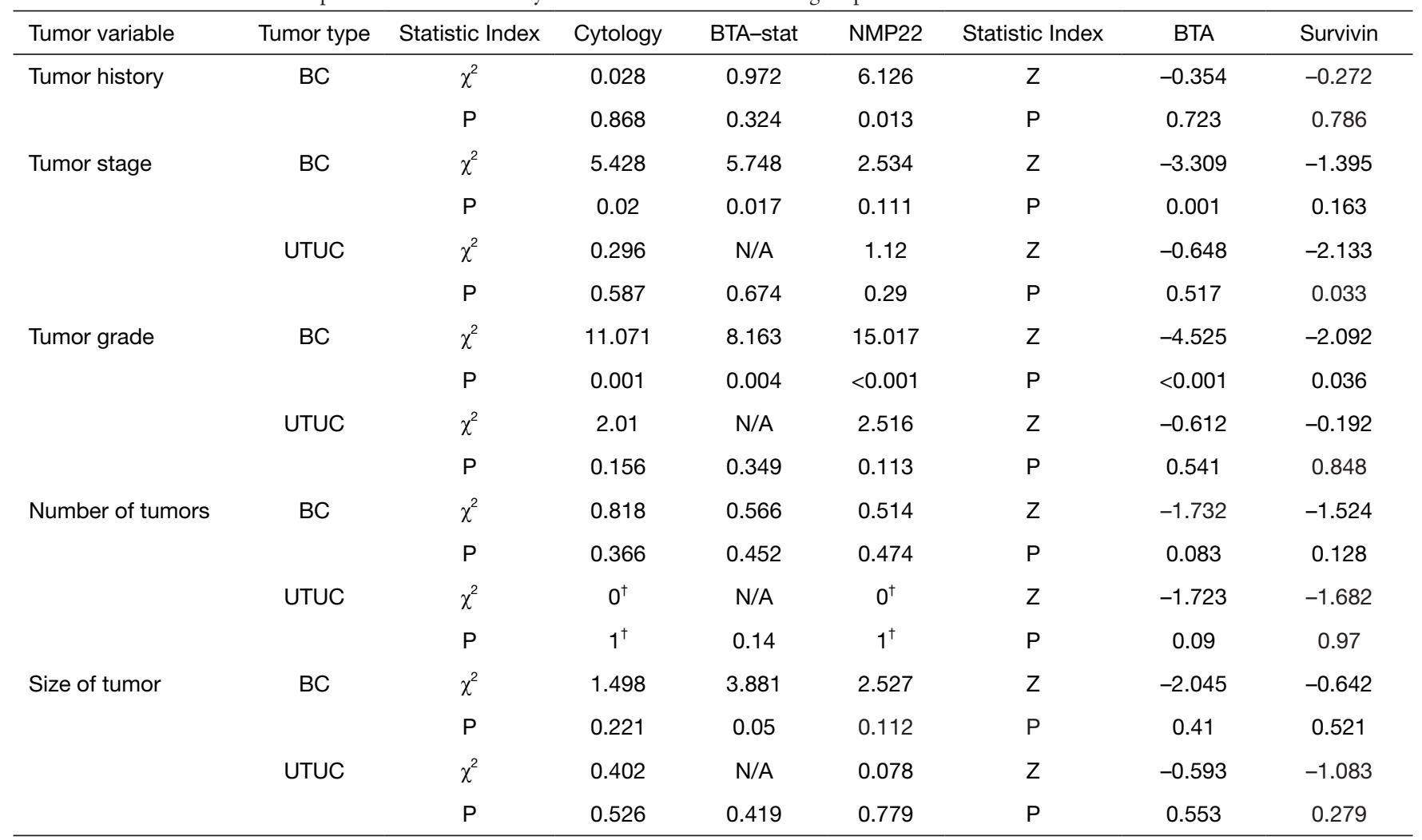

BC, bladder cancer; UTUC, upper-tract urothelial carcinoma. ${ }^{\dagger}$ means this value is infinitely close to 1 or 0 . N/A means that the difference between the two groups of samples is too large to be suitable for analysis 

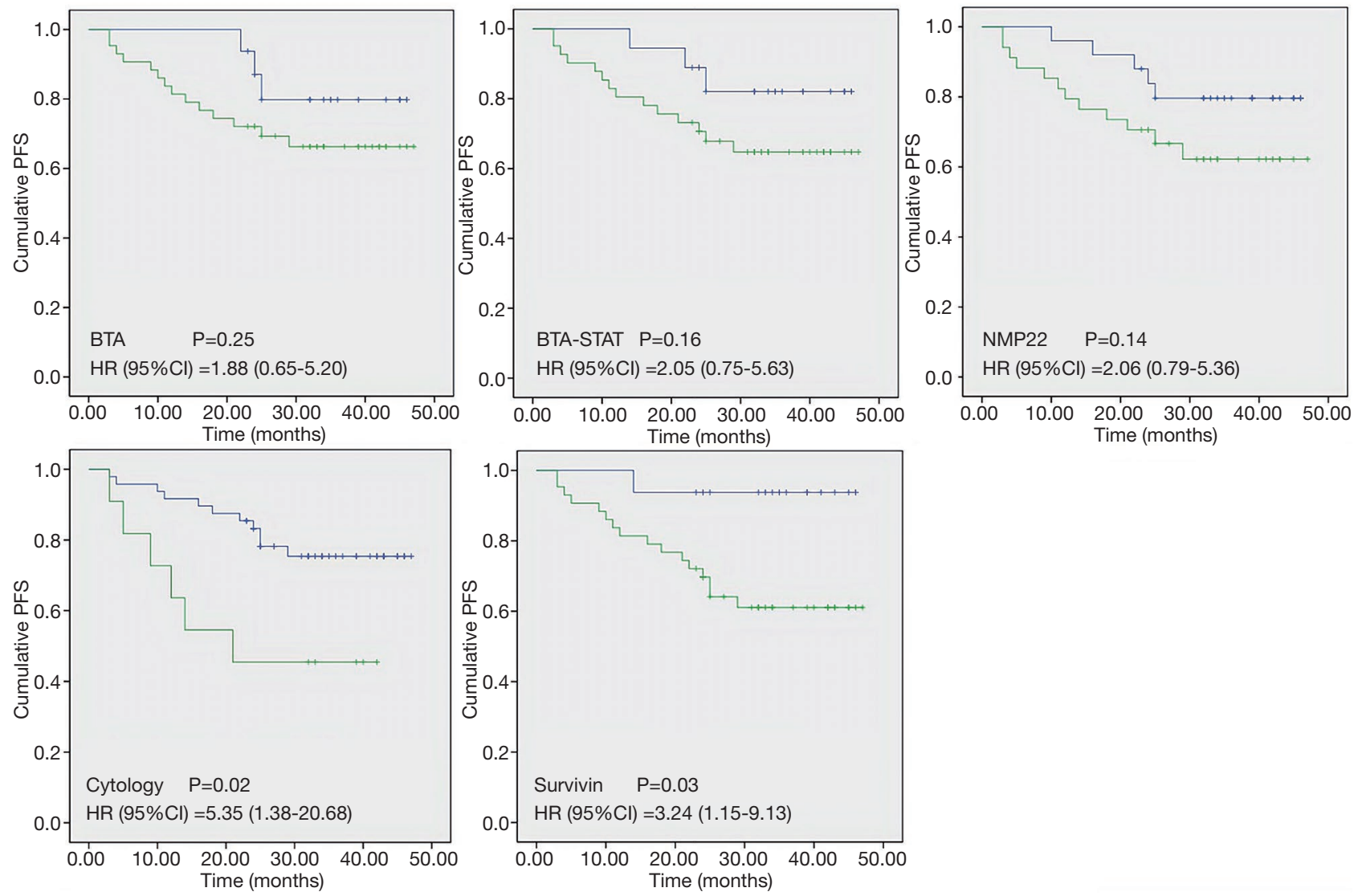

Figure 2 Kaplan-Meier curves for PFS as a function of BTA, BTA-STAT, NMP22, cytology and Survivin in BC. Differences between both groups were calculated by the log-rank test. The green line and blue line represent positive and negative, respectively. Censored cases (+) mark patients that developed no progression during the follow-up or withdraw from the study. PFS, progression-free survival; BC, bladder cancer.

specificity compared to the results obtained by Yafi (8). The BTA quantitative assay had an AUC of 0.84 in the diagnosis of BC, with a sensitivity and specificity of $74 \%$ and $81 \%$, respectively, which were higher than previously reported results $(72 \%, 53 \%)(16)$. In this study, the BTA quantitative detection showed a good diagnostic performance which was consistent with Irani J's findings (17), but contrary to Babjuk's findings that reported that the BTA qualitative detection was more sensitive, while BTA quantitative detection was more specific (18). In the UTUC group, the AUC of the qualitative and quantitative detection of BTA was 0.81 and 0.93 , respectively, which were superior to that seen in bladder cancer.

NMP22 is a protein specific to mitosis and is involved in the distribution of chromatids to daughter cells. The concentration of NMP22 is up to 25 times greater in bladder cancer cell lines than in normal urothelium (8).
This study showed an improved sensitivity with 59\%, and a decreased specificity of 79\% compared to Yifa's findings (19). The diagnostic efficiency in UTUC was better than that in BC with an AUC of 0.76 and a sensitivity of $73 \%$.

Survivin is a member of the inhibitor of apoptosis protein (IAP) family and has a unique role in apoptosis and control of cell division (20). A meta-analysis reported a pooled sensitivity of 0.79 , with a specificity of 0.87 of the Survivin protein test for bladder cancer (21). In this study, the AUC of Survivin for BC was 0.84, with a sensitivity and specificity of $75 \%$ and $83 \%$, respectively. The results were better than Akhtar's findings (22), however the sensitivity of $75 \%$ was slightly lower than the sensitivity of $81 \%$ in this study (23). In the UTUC, Survivin had a better diagnostic performance.

Although the single marker assays are more sensitive than cytology, the latter has a higher specificity. The clinical 

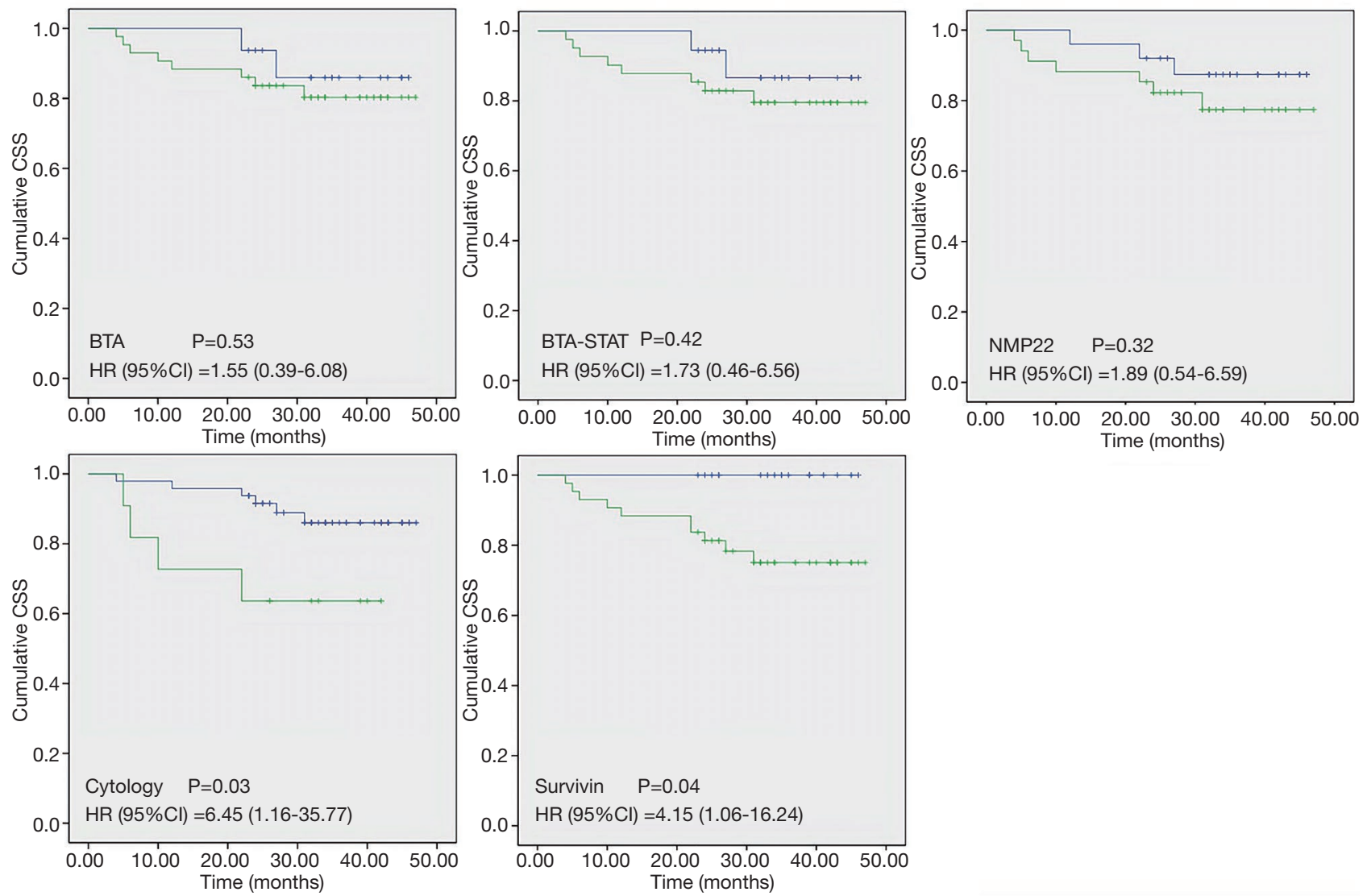

Figure 3 Kaplan-Meier curves for CSS as a function of BTA, BTA-STAT, NMP22, cytology and Survivin in BC. Differences between both groups were calculated by the log-rank test. The green line and blue line represent positive and negative, respectively. Censored cases (+) mark patients that developed no progression during the follow-up or withdraw from the study. CSS, cancer-specific survival; BC, bladder cancer.

application of these tumor markers is limited by false positives. In this study, BTA-stat, BTA, and NMP22 were detected as false positives in urinary tract inflammation and benign prostatic hyperplasia. Tumor markers were negative in 6 cases of patients with haematuria however, hematuria in patients without a malignant disease can result in falsepositive BTA assays (16). Therefore, in-depth research requires a large sample size.

To improve the diagnostic efficacy of tumor markers, combined detection of several tumor markers should be analyzed, which was a useful diagnostic strategy to complement each other and improve the detection value. Several previous studies reported superior efficacy in detection of combining tumor markers compared to the use of single tumor markers $(8,24,25)$. However, a contrary report was given (19). In this study, a triple combination of $\mathrm{S}+\mathrm{B}+\mathrm{C}$ yielded the most valuable combination model with a specificity rate of $97 \%$ and a sensitivity of $86 \%$ and $67 \%$ in UTUC and BC, respectively. In general, the performance of combined detection of tumor markers was evaluated by statistical analysis, however it was hard to using statistical software to determine patient's results of combined tests in clinical work. From our perspective, it was necessary to propose a appropriate recommendation to guide doctors in clinical practice. We suggest that at least the two of Survivin, BTA, and cytology were positive, then the result of the combined detection was considered positive. This recommendation was consistent with our statistical analysis and compensated for the lack of sensitivity of urinary cytology while maintaining high specificity. However, the hypothesis needs more further verification with large sample size.

In this study, there were 49 and 12 cases of primary and recurrent bladder cancer, respectively, and one case of 

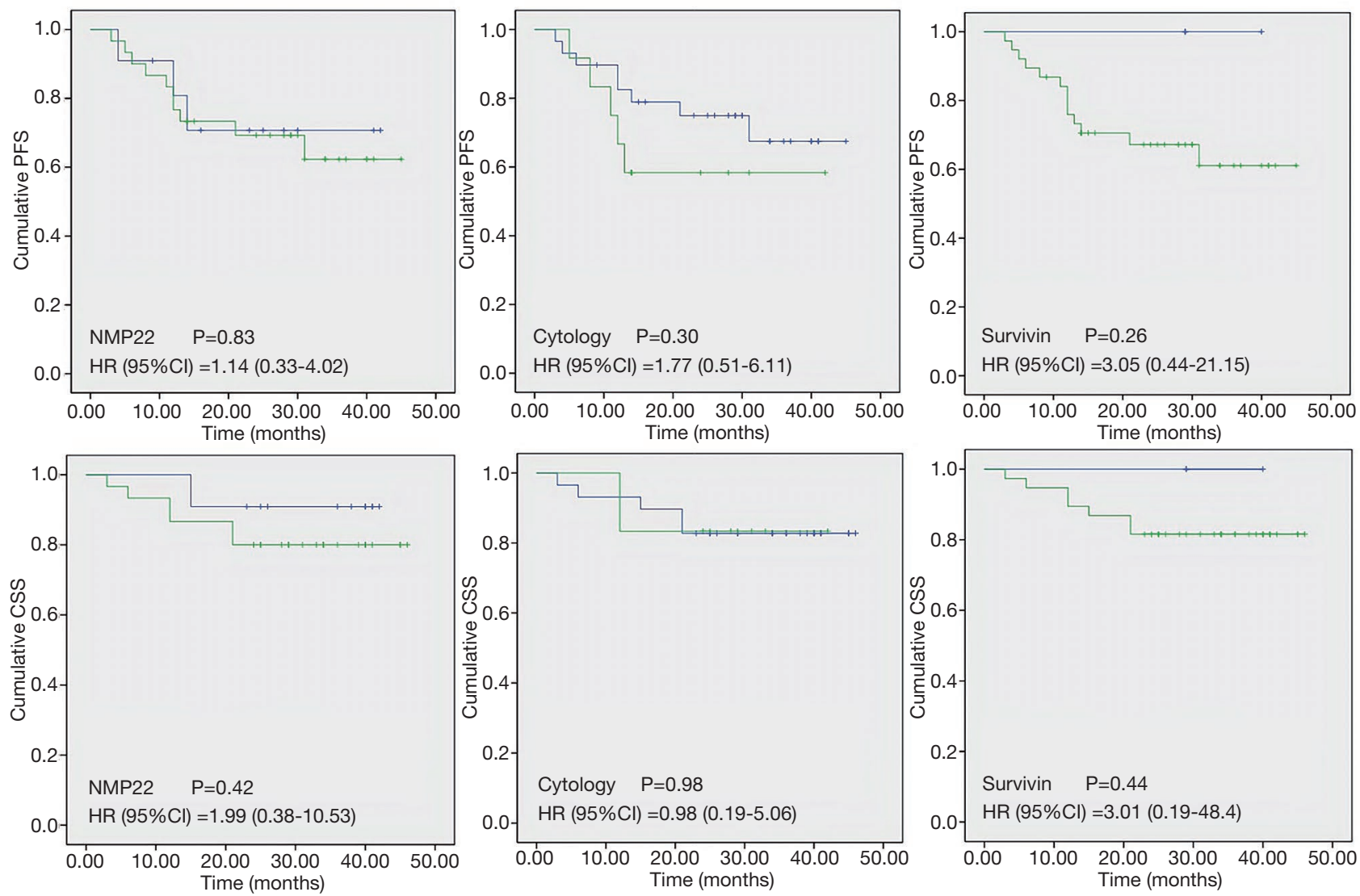

Figure 4 Kaplan-Meier curves for PFS and CSS as a function of the cytology, Survivin and NMP22 in UTUC (the difference of samples between positive group and negative group in both BTA-STAT and BTA are too large to be suitable for analysis). Differences between both groups were calculated by the log-rank test. The green line and blue line represent positive and negative, respectively. Censored cases $(+)$ mark patients that developed no progression during the follow-up or withdraw from the study. PFS, progression-free survival; CSS, cancerspecific survival; UTUC, upper-tract urothelial carcinoma.

recurrence in UTUC. We analyzed the relationship between the tumor markers and the primary and recurrent status of BC. All makers and cytology were no significant correlations with the tumor status except for NMP22 ( $\mathrm{P}=0.013)$. NMP22 was also associated with bladder tumor grade $(\mathrm{P}<0.001)$. According to Yafi's report, NMP22 was associated with stage and grade of $\mathrm{BC}$, but not with other parameters (8), while Jeong's study, NMP22 was only related to the tumor grade (19). This difference might arise as a result of different populations, or the grades and stages of the tumors. Quantitative BTA, and cytology were significantly associated with the stage and grade of BC, while Qualitative BTA-stat was associated with tumor grade and cytology was related to tumor stage (8). The association of the Survivin expression with the histopathological parameters had contradictory viewpoints. In this study, Survivin was not associated with any parameters in BC. In the UTUC group, we observed that Survivin was related to the tumor stage $(\mathrm{P}=0.033)$. None of the urinary markers were associated with the tumor number or size in both BC and UTUC groups.

In the $\mathrm{BC}$ group, histopathological parameters such as a higher tumor grade and an invasive tumor were predictive of progression and survival on univariate analysis. Multivariable analysis revealed that only the tumor grade could predict a shorter PFS and CSS, as earlier reported (26). In the UTUC, tumor grade was shown to have a significant association with the tumor progression by multivariate analysis, which was consistent with other studies $(27,28)$. Previous studies reported that tumor size $>3.0 \mathrm{~cm}$ was a significant predictor of CSS and RFS (29,30), which was not seen in this study.

Considering the role of tumor markers and cytology in tumor progression and survival, $\mathrm{C}(\mathrm{P}=0.01)$ and $\mathrm{S}(\mathrm{P}=0.03)$ 
Table 5 Univariate and multivariate of survival analysis in bladder cancer

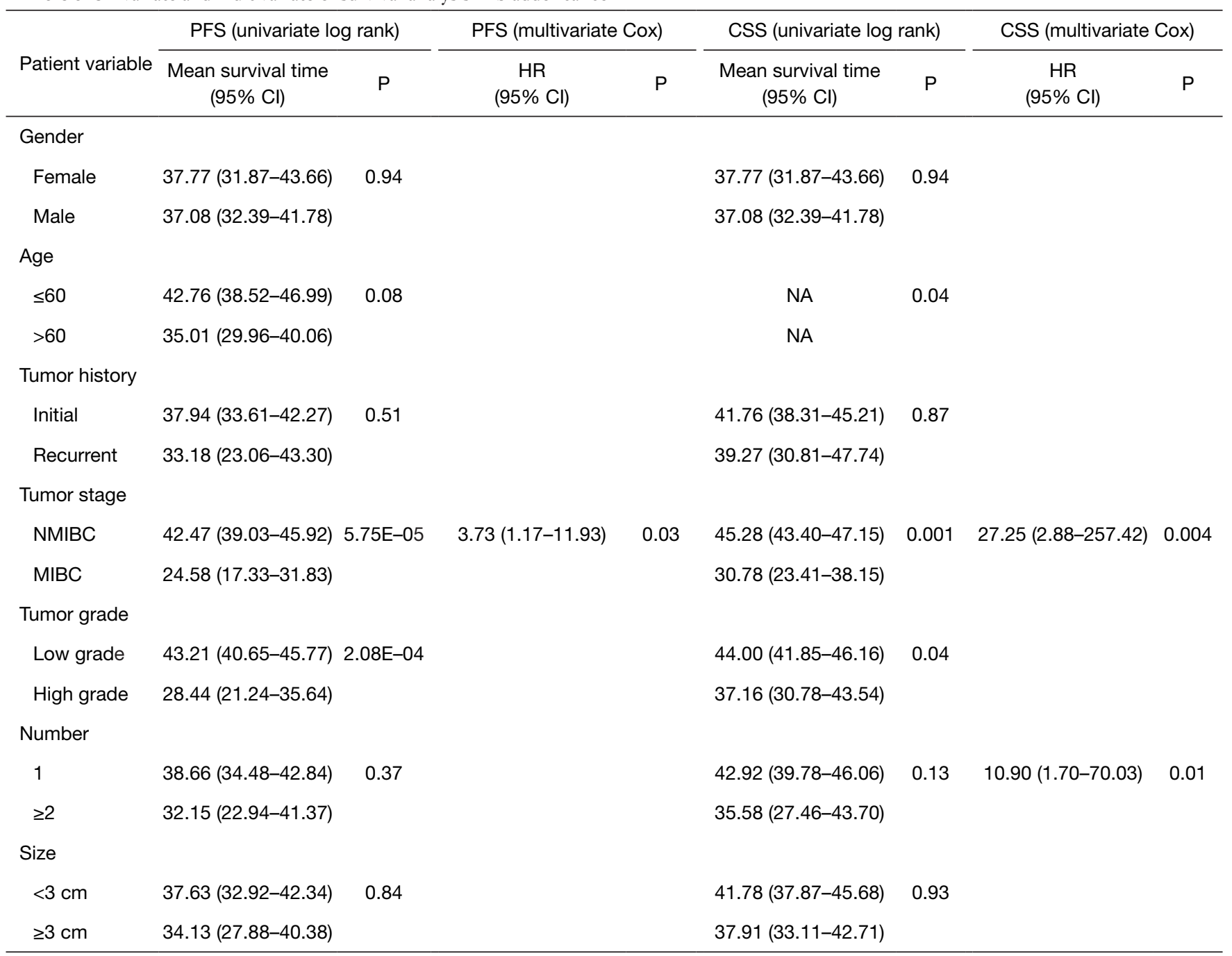

HR, hazard ratio; CI, confidence interval; UTUC, upper-tract urothelial carcinoma; NMIBC, non-muscle-invasive bladder cancer; MIBC, muscle-invasive bladder cancer.

showed significant association with progression, but only the $\mathrm{C}(\mathrm{P}=0.04)$ was statistically associated with CSS in the BC group by using the Kaplan-Meier survival analysis. These results were consistent with these reports (31-33). In the UTUC group, none of the makers had a significant association with the tumor progression and CSS. Among these makers, Survivin was the only maker analyzed in UTUC before, which did not find prognostic value in these studies $(34,35)$, on the contrary, Survivin was considered as an independent prognostic factor (36). A likelihood of falsenegative results may be seen with the small sample size.
Thus a multicenter clinical trial considering more factors in the prognostic analysis in the UC group should be carried out to verify the predictive effect of the biomarkers.

\section{Conclusions}

All biomarkers exhibited diagnostic utility in both BC and UTUC, with a better performance in UTUC. The triple combination $\mathrm{S}+\mathrm{B}+\mathrm{C}$ seems to be the most promising model to serve as a valuable noninvasive method in identifying patients with BC and UTUC. 
Table 6 Univariate and multivariate of survival analysis in UTUC

\begin{tabular}{|c|c|c|c|c|c|c|}
\hline Patient variable & \multicolumn{2}{|l|}{ PFS (univariate log rank) } & \multicolumn{2}{|c|}{ PFS (multivariate Cox) } & \multicolumn{2}{|c|}{ CSS (univariate log rank) } \\
\hline \multicolumn{7}{|l|}{ Gender } \\
\hline Female & $34.39(28.67-40.11)$ & 0.23 & & & $41.57(37.21-45.93)$ & 0.81 \\
\hline Male & $30.94(23.73-38.14)$ & & & & $41.16(37.35-44.96)$ & \\
\hline$\leq 60$ & $40.17(36.89-43.45)$ & 0.07 & & & NA & 0.11 \\
\hline$>60$ & $30.99(24.77-37.21)$ & & & & NA & \\
\hline \multicolumn{7}{|l|}{ Stage } \\
\hline NMIBC & 31.62 (23.90-39.33) & 0.88 & & & $40.15(33.97-46.34)$ & 0.84 \\
\hline Low grade & $39.90(33.30-46.49)$ & 0.06 & $5.86(1.07-32.24)$ & 0.04 & NA & 0.03 \\
\hline High grade & $27.90(21.66-34.14)$ & & & & NA & \\
\hline \multicolumn{7}{|l|}{ Number } \\
\hline 1 & $35.43(30.36-40.50)$ & 0.02 & & & 40.67 (36.69-44.65) & 0.79 \\
\hline$\geq 2$ & $13.60(5.95-21.25)$ & & & & $33.20(21.28-45.12)$ & \\
\hline \multicolumn{7}{|l|}{ Size } \\
\hline$<3 \mathrm{~cm}$ & $35.23(28.06-42.39)$ & 0.57 & & & $39.11(32.95-45.28)$ & 0.48 \\
\hline$\geq 3 \mathrm{~cm}$ & 30.48 (23.98-36.98) & & & & $37.83(33.40-42.25)$ & \\
\hline
\end{tabular}

HR, hazard ratio; Cl, confidence interval; UTUC, upper-tract urothelial carcinoma; NMIBC, non-muscle-invasive bladder cancer; MIBC, muscle-invasive bladder cancer.

\section{Acknowledgments}

We would like to thank Home for Researchers editorial team(www.home-for-researchers.com) for their help in polishing our paper.

Funding: This study was funded by the National Natural Science Foundation of China (82002678), and the Fundamental Research Funds for the Central Universities (lzujbky-2021-kb29).

\section{Footnote}

Reporting Checklist: The authors have completed the REMARK reporting checklist. Available at https://dx.doi. org/10.21037/tcr-21-386

Data Sharing Statement: Available at https://dx.doi. org/10.21037/tcr-21-386
Conflicts of Interest: All authors have completed the ICMJE uniform disclosure form (available at https://dx.doi. org/10.21037/tcr-21-386). The authors have no conflicts of interest to declare.

Ethical Statement: The authors are accountable for all aspects of the work in ensuring that questions related to the accuracy or integrity of any part of the work are appropriately investigated and resolved. The study was conducted in accordance with the Declaration of Helsinki (as revised in 2013). The study was approved by the Ethics Committee of Lanzhou University Second Hospital (approval number: 2016A-058). Written informed consent was obtained from all participants before the samples were collected.

Open Access Statement: This is an Open Access article distributed in accordance with the Creative Commons 
Attribution-NonCommercial-NoDerivs 4.0 International License (CC BY-NC-ND 4.0), which permits the noncommercial replication and distribution of the article with the strict proviso that no changes or edits are made and the original work is properly cited (including links to both the formal publication through the relevant DOI and the license). See: https://creativecommons.org/licenses/by-nc-nd/4.0/.

\section{References}

1. Siegel RL, Miller KD, Jemal A. Cancer statistics, 2016. CA Cancer J Clin 2016;66:7-30.

2. Roupret M, Babjuk M, Comperat E, et al. European Association of Urology Guidelines on Upper Urinary Tract Urothelial Carcinoma: 2017 Update. Eur Urol 2018;73:111-22.

3. Babjuk M, Bohle A, Burger M, et al. EAU Guidelines on Non-Muscle-invasive Urothelial Carcinoma of the Bladder: Update 2016. Eur Urol 2017;71:447-61.

4. Sylvester RJ, van der Meijden AP, Oosterlinck W, et al. Predicting recurrence and progression in individual patients with stage Ta $\mathrm{T} 1$ bladder cancer using EORTC risk tables: a combined analysis of 2596 patients from seven EORTC trials. Eur Urol 2006;49:466-5; discussion 475-7.

5. Green DA, Rink M, Xylinas E, et al. Urothelial carcinoma of the bladder and the upper tract: disparate twins. J Urol 2013;189:1214-21.

6. Sfakianos JP, Cha EK, Iyer G, et al. Genomic Characterization of Upper Tract Urothelial Carcinoma. Eur Urol 2015;68:970-7.

7. Seisen T, Granger B, Colin P, et al. A Systematic Review and Meta-analysis of Clinicopathologic Factors Linked to Intravesical Recurrence After Radical Nephroureterectomy to Treat Upper Tract Urothelial Carcinoma. Eur Urol 2015;67:1122-33.

8. Yafi FA, Brimo F, Steinberg J, et al. Prospective analysis of sensitivity and specificity of urinary cytology and other urinary biomarkers for bladder cancer. Urol Oncol 2015;33:66.e25-31.

9. Favaretto RL, Bahadori A, Mathieu R, et al. Prognostic role of decreased $\mathrm{E}$-cadherin expression in patients with upper tract urothelial carcinoma: a multi-institutional study. World J Urol 2017;35:113-20.

10. Lughezzani G, Burger M, Margulis V, et al. Prognostic factors in upper urinary tract urothelial carcinomas: a comprehensive review of the current literature. Eur Urol 2012;62:100-14.
11. Barkan GA, Wojcik EM, Nayar R, et al. The Paris System for Reporting Urinary Cytology: The Quest to Develop a Standardized Terminology. Adv Anat Pathol 2016;23:193-201.

12. Messer J, Shariat SF, Brien JC, et al. Urinary cytology has a poor performance for predicting invasive or high-grade upper-tract urothelial carcinoma. BJU Int 2011;108:701-5.

13. Reynolds JP, Voss JS, Kipp BR, et al. Comparison of urine cytology and fluorescence in situ hybridization in upper urothelial tract samples. Cancer Cytopathol 2014;122:459-67.

14. Potretzke AM, Knight BA, Vetter JM, et al. Diagnostic Utility of Selective Upper Tract Urinary Cytology: A Systematic Review and Meta-analysis of the Literature. Urology 2016;96:35-43.

15. Kinders R, Jones T, Root R, et al. Complement factor $H$ or a related protein is a marker for transitional cell cancer of the bladder. Clin Cancer Res 1998;4:2511-20.

16. Miyake M, Goodison S, Rizwani W, et al. Urinary BTA: indicator of bladder cancer or of hematuria. World J Urol 2012;30:869-73.

17. Irani J, Desgrandchamps F, Millet C, et al. BTA stat and BTA TRAK: A comparative evaluation of urine testing for the diagnosis of transitional cell carcinoma of the bladder. Eur Urol 1999;35:89-92.

18. Babjuk M, Kostirova M, Mudra K, et al. Qualitative and quantitative detection of urinary human complement factor H-related protein (BTA stat and BTA TRAK) and fragments of cytokeratins 8, 18 (UBC rapid and UBC IRMA) as markers for transitional cell carcinoma of the bladder. Eur Urol 2002;41:34-9.

19. Jeong S, Park Y, Cho Y, et al. Diagnostic values of urine CYFRA21-1, NMP22, UBC, and FDP for the detection of bladder cancer. Clin Chim Acta 2012;414:93-100.

20. Akhtar M, Gallagher L, Rohan S. Survivin: role in diagnosis, prognosis, and treatment of bladder cancer. Adv Anat Pathol 2006;13:122-6.

21. Liang Z, Xin R, Yu Y, et al. Diagnostic value of urinary survivin as a biomarker for bladder cancer: a systematic review and meta-analysis of published studies. world $\mathrm{J}$ Urol 2018;36:1373-81.

22. Schmidt J, Propping C, Siow WY, et al. Diagnostic and prognostic value of bladder cancer-related transcript markers in urine. J Cancer Res Clin Oncol 2016;142:401-14.

23. Srivastava AK, Singh PK, Srivastava K, et al. Diagnostic role of survivin in urinary bladder cancer. Asian Pac J Cancer Prev 2013;14:81-5. 
24. Rosser CJ, Ross S, Chang M, et al. Multiplex protein signature for the detection of bladder cancer in voided urine samples. J Urol 2013;190:2257-62.

25. Valenberg F, Hiar AM, Wallace E, et al. Prospective Validation of an mRNA-based Urine Test for Surveillance of Patients with Bladder Cancer. Eur Urol 2019;75:853-60.

26. Chan OTM, Furuya H, Pagano I, et al. Association of MMP-2, RB and PAI-1 with decreased recurrence-free survival and overall survival in bladder cancer patients. Oncotarget 2017;8:99707-21.

27. Wu Y, Dong Q, Liu L, et al. The impact of tumor location and multifocality on prognosis for patients with upper tract urothelial carcinoma: a meta-analysis. Sci Rep 2014;4:6361.

28. Chromecki TF, Cha EK, Fajkovic H, et al. The impact of tumor multifocality on outcomes in patients treated with radical nephroureterectomy. Eur Urol 2012;61:245-53.

29. Shibing Y, Liangren L, Qiang W, et al. Impact of tumour size on prognosis of upper urinary tract urothelial carcinoma after radical nephroureterectomy: a multi-institutional analysis of 795 cases. BJU Int 2016;118:902-10.

30. Espiritu PN, Sverrisson EF, Sexton WJ, et al. Effect of tumor size on recurrence-free survival of upper tract

Cite this article as: Gong YW, Wang YR, Fan GR, Niu Q, Zhao YL, Wang H, Svatek R, Rodriguez R, Wang ZP. Diagnostic and prognostic role of BTA, NMP22, survivin and cytology in urothelial carcinoma. Transl Cancer Res 2021;10(7):3192-3205. doi: 10.21037/tcr-21-386 urothelial carcinoma following surgical resection. Urol Oncol 2014;32:619-24.

31. Bell MD, Yafi FA, Brimo F, et al. Prognostic value of urinary cytology and other biomarkers for recurrence and progression in bladder cancer: a prospective study. World J Urol 2016;34:1405-9.

32. Ku JH, Kwak C, Lee HS, et al. Expression of survivin, a novel inhibitor of apoptosis, in superficial transitional cell carcinoma of the bladder. J Urol 2004;171:631-5.

33. Shariat SF, Ashfaq R, Karakiewicz PI, et al. Survivin expression is associated with bladder cancer presence, stage, progression, and mortality. Cancer 2007;109:1106-13.

34. Mathieu R, Klatte T, Margulis V, et al. Survivin is not an independent prognostic factor for patients with upper tract urothelial carcinoma: a multi-institutional study. Urol Oncol 2015;33:495.e15-22.

35. Fromont G, Roupret M, Amira N, et al. Tissue microarray analysis of the prognostic value of E-cadherin, Ki67, p53, p27, survivin and MSH2 expression in upper urinary tract transitional cell carcinoma. Eur Urol 2005;48:764-70.

36. Jeong IG, Kim SH, Jeon HG, et al. Prognostic value of apoptosis-related markers in urothelial cancer of the upper urinary tract. Hum Pathol 2009;40:668-77. 